\title{
TH1F-(5)-6
}

\section{D Four- Three- Two-Photon and Multi-Harmonic Microscopy of Lateral-Over-Grown GaN}

\author{
Chien-Hung Tseng, Shi-Wei Chu, and Chi-Kuang Sun \\ Graduate Institute of Electro-Optical Engineering, National Taiwan University, Taipei, 10617 \\ TAIWAN, R.O.C. \\ Tel: +886-2-23635251 ext. 406. FAX: +886-2-23677467. Email: ptchien@ms4.hinet.net
}

\author{
Paul Fini and Steven P. DenBaars \\ Materials Department and Electrical and Computer Engineering Department, College of \\ Engineering, \\ University of California, Santa Barbara, Santa Barbara, California 93106
}

\begin{abstract}
Complete information regarding the distribution of growth quality, defect state, and piezoelectric field in lateral-over-grown GaN can be obtained simultaneously through multi-photon (4 and 3) and multi-harmonic (3 and 2) microscopy.
\end{abstract}

Taking advantage of strong 4-photon absorption of $1 \mathrm{eV}$ light in $\mathrm{GaN}$ samples, we demonstrated the first ever 4-photon microscopy using GaN material system. Combining with 3-photon fluorescence and second and third harmonic generation microscopies, we studied a lateral overgrown $\mathrm{GaN}$ sample with high $3 \mathrm{D}$ resolution. Complete information regarding the distribution of growth quality, defect state, and piezoelectric field in lateral-over-grown GaN can be obtained simultaneously through multi-photon (4 and 3$)$ and multi-harmonic (3 and 2) microscopy based on a 1230-nm femtosecond Cr:forsterite laser.

The samples under study include a bulk $\mathrm{GaN}$ layer with high quality coalesced lateral epitaxial overgrowth (LEO) [1].

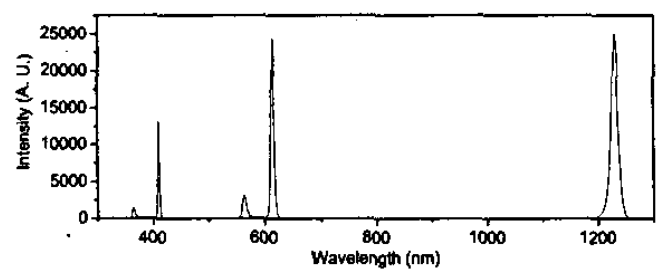

Fig. 1 The nonlinear emission spectrum from a bulk $\mathrm{GaN}$ excited by a single $\mathrm{Cr}$ :forsterite laser.
(A)

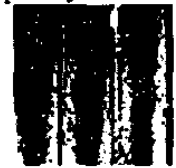

(B)

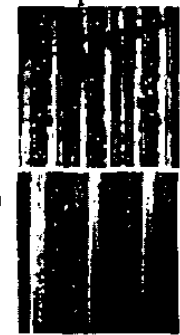

Fig. 2 Plan-view scanning microscopic images corresponding to (A) edge luminescence, (B) yellow luminescence, (C) SHG and

(D) THG in a laterally overgrown bulk GaN. Image size: $60 \times 60-\mu \mathrm{m}^{2}$.

Fig. 1 is the nonlinear emission spectrum from the bulk GaN excited by a single femtosecond $\mathrm{Cr}$ :forsterite laser centered at $1230 \mathrm{~nm}$. Fig. 2A-2D are plan-view sectioned scanning microscopic images of these nonlinear signals in a LEO bulk GaN. The images were formed by moving the sample perpendicular to laser focusing direction and recording spectrum at each point. The bright bandedge emission at $365 \mathrm{~nm}$ corresponding to good GaN edge emission quality in regions above the $15-\mu \mathrm{m}$-wide $\mathrm{SiO}_{2}$ stripes suggests the $\mathrm{SiO}_{2}$ stripes' ability to block the threading effects. On the other hand, the defect-state-related yellow fluorescence (563 nm) is strongest at the edge of $\mathrm{SiO}_{2}$ stripes indicating higher deep-acceptor-state density there (Fig. 2B). And strong SHG signal at $615 \mathrm{~nm}$ of $\mathrm{GaN}$ grown above $\mathrm{SiO}_{2}$-stripe openings shows the large piezoelectric field induced by unrelaxed strain (Fig. 2C). Moreover, the contrast of THG in Fig. 2D might reveal the interfaces inside the samples and distribution of bandtail states due to their close resonance.

In summary, taking advantage of strong 4-photon absorption of $1 \mathrm{eV}$ light in GaN samples, we demonstrated a new approach to mapping LEO GaN structural information combining multi-photon fluorescence (4 and 3 ) and multi-harmonic ( 3 and 2) scanning microscopy which provides not only superior 3D (better than $500 \mathrm{~nm}$ ) resolution but also plentiful information of $\mathrm{GaN}$ including growth quality (edge PL), defect state distribution (yellow luminescence), piezoelectric field (SHG) and refractive index change. This study also represents the first ever 4-photon microscopy in history.

\section{REFERENCES}

[1] See P. Fini et al., "High-quality coalescence of laterally overgrown GaN stripes on GaN/sapphire seed layers" Appl. Phys. Lett., 75, 1706-1708 (1999), and the references in it. 